\title{
Enhanced firefly optimizer with deep neural network for the detection of epileptic seizures using EEG signals
}

\author{
Ruchi Sharma, Khyati Chopra
}

\begin{abstract}
Currently, Electroencephalogram (EEG) is extensively used for diagnosing the epilepsy. The objective of this research is to investigate the changes in epilepsy frequency by proposing a new optimization based deep learning model. At first, the EEG recordings were acquired from two online databases; Bern Barcelona (BB), and Bonn University (BU). Then, Chebyshev type two filter was implemented to remove the unwanted artifacts from the acquired EEG signals. Further, Multivariate Variational Mode Decomposition (MVMD) methodology was applied to decompose the denoised EEG signals. The signal decomposition helps in finding the necessary information, which required to model the complex time series data. Then, the features were extracted from decomposed signals by using fifteen entropy, linear and statistical features. In addition, enhanced firefly optimization technique was proposed for optimizing the extracted features. In the enhanced firefly optimizer, a crossover operator of genetic algorithm was added for enhancing the local convergence rate that gives better classification. At last, the optimized feature vectors were classified by Deep Neural Network (DNN) that includes two circumstances (seizure and healthy), and (Interictal, ictal, and normal). From the experimental simulation, the proposed model improvement maximum of $1.4 \%$, and $8.82 \%$ of accuracy in $B U$ and BB EEG datasets, respectively related to the existing models. Index values: Chebyshev type two filter, deep neural network, enhanced firefly optimization, epileptic seizure detection, and multivariate variational mode decomposition.
\end{abstract}

\section{INTRODUCTION}

In neurological disorder, epilepsy is a challenging subject, which gained more attention among the researchers and affects nearly $2 \%$ of the world population [1-2]. The epilepsy leads to loss of consciousness, physical variation in the movements, strange emotions, muscle spasms and death [3]. So, it is essential to detect the epilepsy at early stage for reducing the death rate. Epilepsy is characterized by recurrent seizures that results from the extreme discharges of the brain cells [4-6]. For epilepsy recognition, the EEG signals are widely utilized for investigating the brain activities [7]. EEG is a non-invasive technique that significantly estimate the neuronal and electrical activities in high temporal resolution [8-9]. The other imaging techniques, which are used for inspecting the brain activities are Magnetic Resonance Imaging (MRI), Functional MRI (FMRI), computed tomography, positron emission tomography, etc. The visual evaluation of EEG signals results in human errors and time consuming [10-12].

Revised Manuscript Received on May 15, 2020.

* Correspondence Author

Ruchi Sharma*, Research Scholar GD Goenka University Gurugram,India

Khyati Chopra, Department of Elec.and Electronics Engineering GD Goenka University Gurugram, India

(C) The Authors. Published by Blue Eyes Intelligence Engineering and Sciences Publication (BEIESP). This is an open access article under the CC BY-NC-ND license (http://creativecommons.org/licenses/by-nc-nd/4.0/)
To address these concerns and also to improve the epilepsy detection, several automated systems are developed in the past studies such as $l_{1}$ penalized regression [13], Least Squares Support Vector Machine (LSSVM) [14], neural mass mode [15], artificial neural network [16], SVM [1718], kernel principal component analysis [19], long short term memory [20], etc. In the conventional approaches, it is difficult to identify the suitable EEG representation such that the non-epileptic patterns are differentiable from epileptic patterns. To overcome this issue, a new optimization based model is proposed for enhancing the performance of epilepsy recognition. Initially, the EEG recordings were acquired from BB EEG and BU EEG databases. Then, Chebyshev type 2 filter was implemented to remove the artifacts (eye movements) from the acquired signals. Compared to other filtering techniques, Chebyshev type 2 filter has sharp transition between the stopband and the passband lower order filter that results in fast execution speed and limited absoluter error. The denoised EEG signals were decomposed by employing MVMD in order to analyse the subtle changes in frequency. Then, entropy, linear and statistical features were combined to extract the feature vectors from the decomposed signals. Further, the extracted features were optimized using enhanced firefly optimizer. A crossover operator of genetic algorithm was included in firefly algorithm for improving the local convergence rate that results in better classification rate. The optimized feature vectors were classified by using DNN classifier that includes two cases (seizure and healthy), and (Interictal, ictal, and normal). Compared to other classifiers, DNN effectively utilizes the resources and also allows time cycle reduction. In the result and discussion section, the proposed model performance was evaluated by means of accuracy, Positive Predictive Value (PPV), specificity and sensitivity.

A few research papers are surveyed in the section 2. The explanation about the proposed model is detailed in the section 3. In section 4, the experimental consequences are detailed with tabular and graphical representation. Section 5 indicates the conclusion of the present study.

\section{LITERATURE SURVEY}

N. Mahmoodian, et al, [21] used cross bispectrum features for recognizing epilepsy in EEG dataset. The cross bispectrum was a non-linear and multivariate methodology, which was used for identifying the relationship between dissimilar frequencies from an individual channel. Then, the combination of non-linear and linear features were applied for extracting the feature vectors from the collected signals. The extracted features were classified by employing SVM. In this literature study,

Blue Eyes Intelligence Engineering 
Freiburg iEEG database was used for verifying the effectiveness of the developed system.

From the experimental consequence, the developed system attained superior performance in epilepsy detection related to other models in light of accuracy, specificity and sensitivity. However, SVM classifier supports only binary classification, which was considered as a major concern in this study. K. Jindal, et al, [22] implemented Tunable Q wavelet Transform (TQWT) for epilepsy recognition on the basis of non-linear features. In this study, BU EEG database was undertaken for experimental investigation that includes three classes such as seizure, non-seizure and pre-seizure. In this study, the developed model includes three phases; decomposition, extraction of feature, and classification. After data collection, TQWT was utilized to decompose the signals into time frequency bands. Then, approximate entropy, Katz's, and Higuchi's fractal dimension were applied to extract the feature vectors, which were classified by employing random forest and SVM classifier in the third phase. From the experimental investigation, it was confirmed that the developed model attained better performance in epilepsy recognition. In large dataset, the random forest leads to data imbalance that may results in poor classification performance. H. Peng, et al, [23] developed Dictionary Learning with Homotopy (DLWH) approach on the basis of sparse representation for epilepsy recognition. Initially, homotopy approach was utilized for attaining the sparse representation of EEG coefficients. Then, $l_{1}$ normalization was applied to determine the reconstruction error between the non-epileptic and epileptic samples. In this research, the developed approach performance was verified on two databases; BU EEG and Children's Hospital Boston-Massachusetts Institute of Technology (CHB-MIT) database. In the experimental phase, the developed approach showed good performance in classification that includes two cases (seizure and healthy), and (Interictal, ictal, and normal). The developed approach required more manual intervention that was considered as a major concern.

G. Wang, et al, [24] developed Partial Directed Coherence Analysis (PDCA) to recognize the epilepsy patients. Initially, the original EEG signals were collected from BU EEG database. Then, autoregressive features were applied for extracting the feature vectors from the collected EEG signals. In addition, sum-up the intensity propagated information for attaining the outflow information related to EEG channel that significantly lessen the dimensionality of the extracted feature vectors, Finally, the non-seizure and seizure signals were classified by employing SVM classifier. The developed model almost achieved better accuracy in BU EEG database related to the prior models. The SVM includes many key parameters for achieving better classification that need to be set correctly, or else it may leads to poor epilepsy classification.

A. Bhattacharyya, et al, [25] developed 2D reconstructed phase space with LSSVM for epilepsy recognition. In this literature, the original EEG signals were acquired from BB EEG database. Then, empirical wavelet transform was utilized for decomposing the signals into time frequency bands. In the next phase, reconstructed phase space was applied for extracting the non-linear feature vectors from the decomposed signals. Finally, the LSSVM classifier was utilized for classifying the interictal, ictal and normal signals for early recognition of epilepsy. The developed model effectively identifies the epileptic zone that helps the clinicians in curing the epilepsy. While classifying the data, the LSSVM classifier includes two concerns such as no interpretability and easy occurrence of over fitting. A new optimization based model is proposed for enhancing the performance of epilepsy recognition in this research article.

\section{PROPOSED MODEL}

The detailed explanation about the proposed model is given this section. The proposed model includes six phases; Signal collection: BB and BU EEG databases, Signal denoising: Chebyshev type 2 filter, Signal decomposition: MVMD, Feature extraction: combination of entropy, linear and statistical features, Feature optimization: Enhanced firefly optimizer, and Classification: DNN. The work flow of proposed model is indicated in figure 1.

\subsection{Dataset description}

In this section, the description about the undertaken databases (BB EEG and BU EEG) is explained. The BB EEG database comprises of two classes; non-focal and focal signals [26]. Each class includes 3750 segments (10240 data points per segment) under a sampling rate of $512 \mathrm{~Hz}$ and with a duration 20 seconds. The sample collected BB EEG signals are graphically specified in figure 2 .

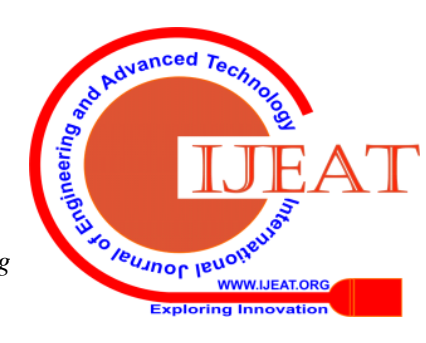




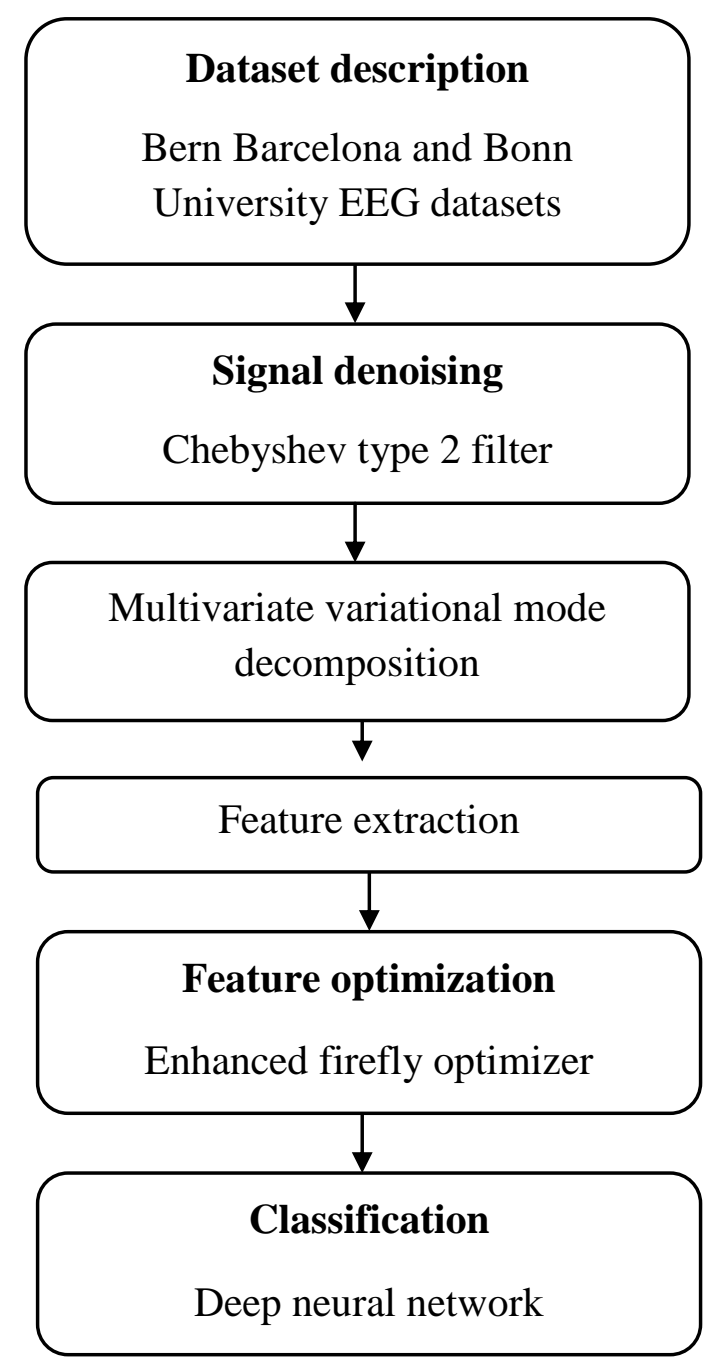

Figure 1. Flow chart of the proposed model

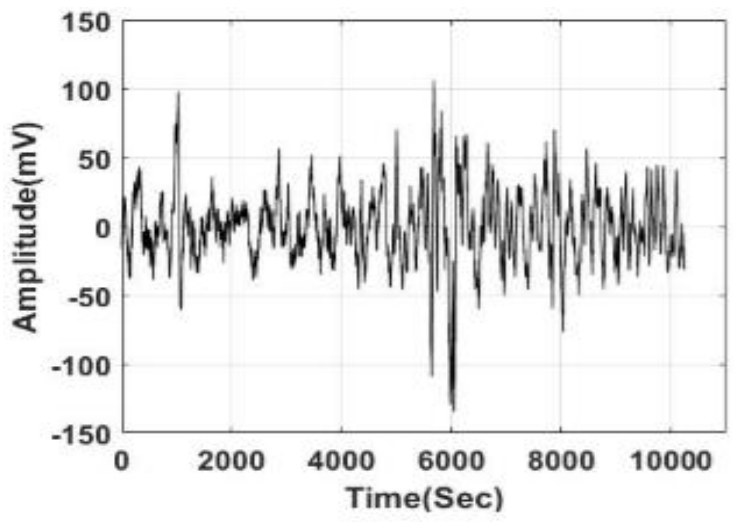

(a)

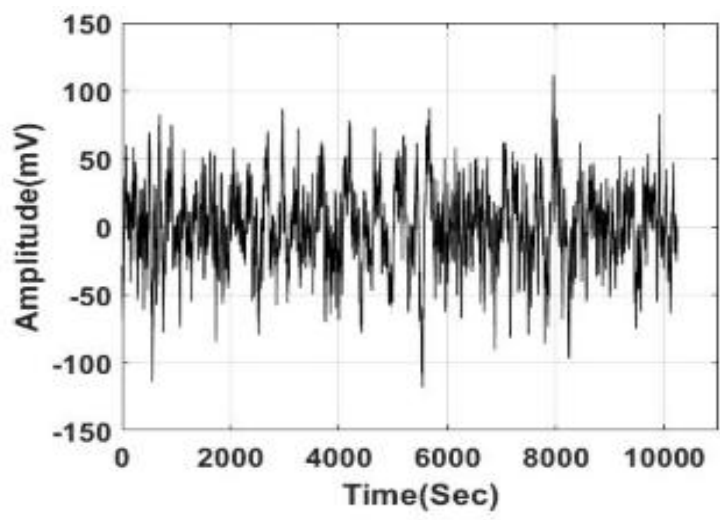

(b)

Figure 2. BB EEG dataset; a) Focal signal, and b) Non-focal signal

The BU EEG database consists of five EEG sub-sets such as F, N, O, S, and Z for both epileptic and healthy subjects [2728]. Every subset consists of 100 EEG segments, which are partitioned from single channel recording. Each EEG segment consists of 4096 sampling points with the duration of 23.06 seconds. After visually investigating the artifacts occurred by muscle activity, the EEG segments are cut-out and chosen from multichannel EEG recordings. During seizure, the sub-set " $\mathrm{S}$ " is recorded that contains only ictal movement. During free interval, the sub-set " $F$ " is recorded with in the epileptogenic zone.

\& Sciences Publication C) Copyriaht: All riahts reserved.

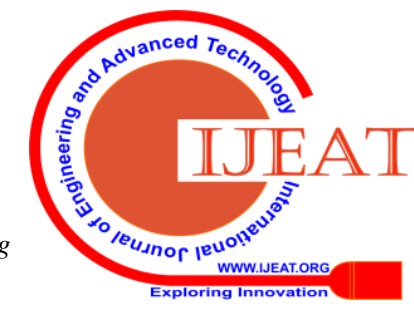


For 5 healthy subjects, the sub-sets " $O$ " and " $Z$ " are recorded with eyes open and closed. In addition, the sub-set " $\mathrm{N}$ " is recorded from hippocampal formation of the brain.

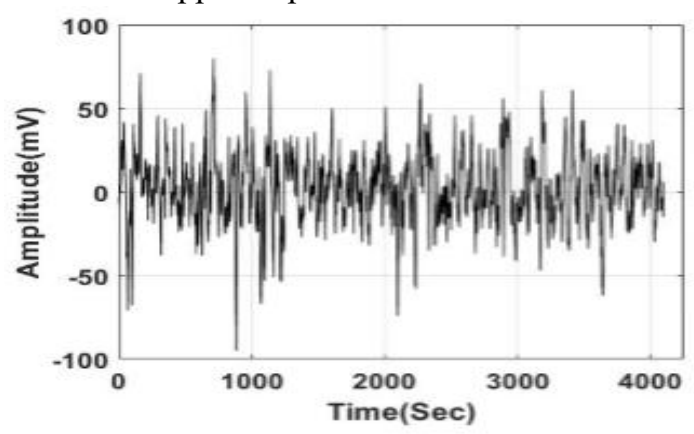

(a)
The EEG signals are recorded with a 128 channel amplifier at a sampling rate of $173 \mathrm{~Hz}$. The sample collected BU EEG signals are graphically indicated in figure 3.

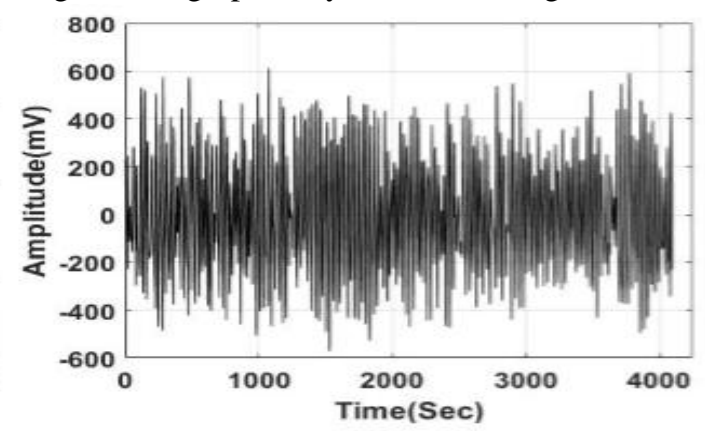

(b)

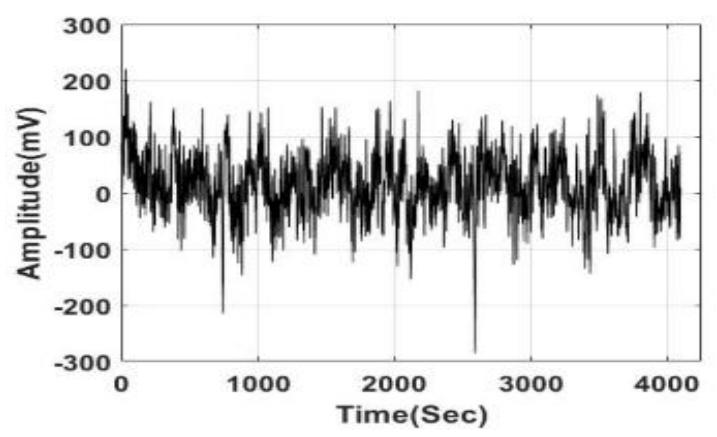

(c)

Figure 3. BU EEG database; a) Ictal signal, b) Interictal signal, and c) Normal signal

\subsection{Signal denoising and decomposition}

After acquiring the EEG signals, Chebyshev type 2 filter was implemented for eliminating the unwanted noises (muscle and eye movements) from the acquired signals. The Chebyshev type 2 filter does not rolloff, so it requires several components. In the stopband, it has equiripple but the Chebyshev type 2 filter has no ripple in the passband. Related to other filtering methods, the undertaken filter has fast execution speed and limited absoluter error. The Chebyshev type 2 filter formula is indicated in equation (1).

Chebyshev type 2 filter $=$

$$
\frac{1}{\sqrt{1+\rho^{2} T_{n}^{2}\left(\frac{w}{w_{0}}\right)}}
$$

Where, $T_{n}$ is stated as Chebyshev polynomial, $\rho$ is denoted as ripple factor, and $w_{0}$ is indicated as cut-off frequency, where the range is $60 \mathrm{~Hz}$.

Then, the MVMD technique is implemented to decompose the denoised signals, which is the extension of VMD. The objective of MVMD is used to extract the predetermined number of multivariate modulated oscillations $u_{k}(t)$ from the denoised signals $x(t)$ which includes $n$ number of channels $C, \quad x(t)=\left[x_{1}(t), \ldots x_{C}(t)\right]$. The mathematical representation of $x(t)$ is indicated in equation (2).

(2)

$$
x(t)=\sum_{k=1}^{K} u_{k}(t)
$$

Where, $u_{k}(t)=\left[u_{1}(t), \ldots u_{C}(t)\right]$. To extract $u_{k}(t)$, the vector analytic representation of $u_{k}(t)$ is changed as $u_{+}^{k}(t)$ [29]. The $u_{k}(t)$ bandwidth is calculated by considering $l_{2}$ normalization of the gradient function $u_{+}^{k}(t)$. The resultant cost function $f$ of MVMD is utilized in the VMD optimization problem that is mathematically defined in equation (3).

$$
f=\sum_{k}\left\|\partial_{t}\left[e^{-j w k^{t}} u_{+}^{k}(t)\right]\right\|_{2}^{2}
$$

Where, $\partial_{t}$ is indicated as partial derivative operation, $w_{k}$ is denoted as center frequency of $k$ modes, and $u_{k}$ is specified as number of modes. The component $w_{k}$ is utilized in the harmonic mixing of vector $u_{+}^{k}(t)$. Hence, the modulated multivariate oscillation bandwidth is calculated by shifting the unilateral frequency spectrum of $u_{+}^{k}(t)$ that updates the cost function $f$ as stated in equation (4).

$f=\sum_{k} \sum_{c}\left\|\partial_{t}\left[u_{+}^{k, c}(t) e^{-j w k^{t}}\right]\right\|_{2}^{2}$

(4)

Where, $u_{+}^{k, c}$ is indicated as complex valued signal with signal component in equation (4). The constrained optimization issue of MVMD is indicated in equation (5).

$\underset{\left\{u_{k, c}\right\},\left\{w_{k}\right\}}{\operatorname{minimize}} \sum_{k} \sum_{c}\left\|\partial_{t}\left[u_{+}^{k, c}(t) e^{-j w k^{t}}\right]\right\|_{2}^{2}$

In MVMD technique, the liner equality constrains are equal to the number of channel $C$. The sample decomposed signal is graphically indicated in figure 4 .

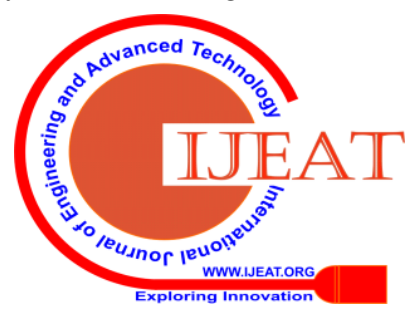




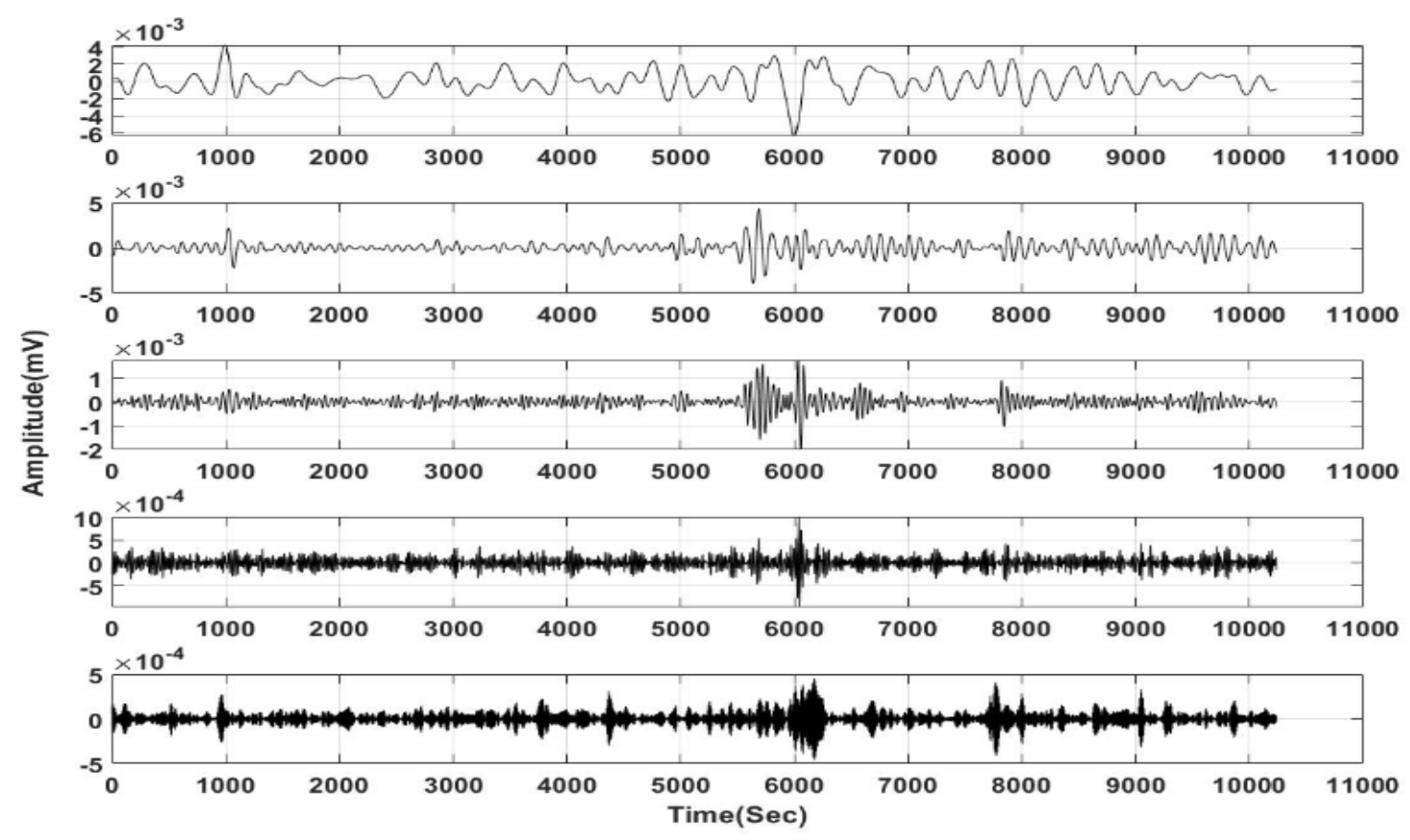

Figure 4. Sample decomposed EEG signal

\subsection{Feature extraction and optimization}

After decomposing the EEG signals, feature extraction is performed by using statistical features: root sum square, moment, skewness, minimum, kurtosis, mean, maximum, standard deviation, and peak root mean square, Entropy features: tsallis entropy and Renyi entropy, Linear features: spectral flux, spectral flatness, mean frequency, and cumulative features. The major benefit of combining more features results in effective occlusion and clutter. Then, the extracted feature vectors are given as the input to enhanced firefly optimizer for optimizing or selecting the active features. Generally, the firefly optimization algorithm is developed on the basis of firefly characteristics. The traditional firefly optimizer includes three basic rules,

- The fireflies are unisex, so a firefly is attracted to another firefly based on their sex.

- The light intensity or brightness of a firefly is identified based on the objective function.

- Brightness is inversely proportional to the attractiveness, so the less bright firefly moves towards high bright firefly. If no firefly is brighter, then the fireflies moves randomly.

The firefly $\beta$ attractiveness is mathematically denoted in equation (6).

$$
\beta=\beta_{0} e^{-\gamma r_{i j}^{2}}
$$

Where, $r_{i j}$ is represented as distance between the fireflies $i$ and $j$, which is measured by using Cartesian distance measure, $\gamma$ is indicated as coefficient of light absorption, and $\beta_{0}$ is specified as attractiveness at $r_{i j}=0$. Equation (7) denotes the movement of attractiveness between the fireflies $i$ and $j$.

$$
x_{i}=x_{i}+\Delta x \text {, where } \Delta x=
$$

$\beta\left(x_{j}-x_{i}\right)+\alpha \epsilon_{i}$
Where, $\epsilon_{i}$ is indicated as random value, $x_{i}$ is represented as firefly position, and $\alpha$ is stated as randomization parameter that is defined in equation (8).

$$
\alpha(t)=\alpha(0)\left(\frac{10^{-4}}{0.9}\right)^{t / t_{\max }}
$$

Where, $t$ is represented as iteration number. Then, the mutation and uniform crossover genetic operators are applied in the fireflies for improving the local convergence rate. In this research, the mutation probability is set as $30 \%$ and the crossover probability is set as $100 \%$. Hence, the chromosomes in the genetic algorithm are considered as fireflies. Finally, the selected feature vectors are classified by employing DNN classifier. The enhanced firefly method is graphically indicated figure 5 .

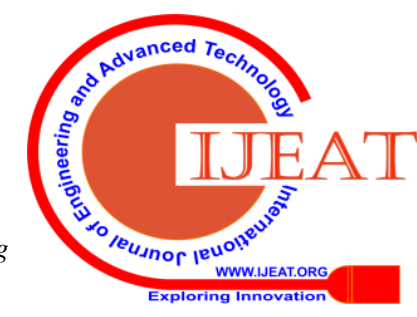


Enhanced firefly optimizer with deep neural network for the detection of epileptic seizures using EEG signals

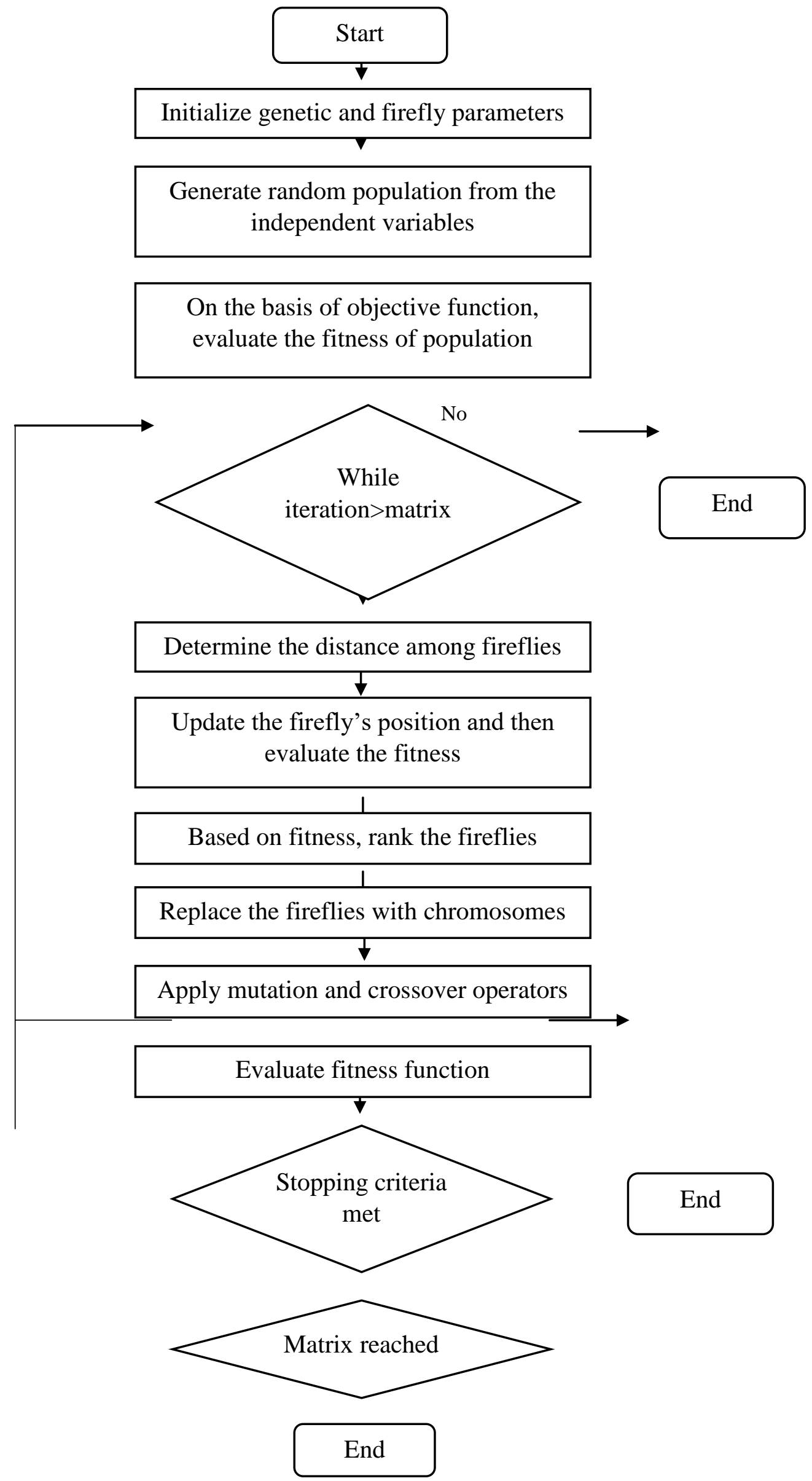

Figure 5. Work flow of enhanced firefly 


\subsection{Classification}

The DNN is a feed forward network that contains multiple transformation layers and non-linearity with the output of every layer which feeds into the succeeding layers. The DNN model is mathematically presented in the equations (9) and (10).

$$
Z^{(l)}=y^{(l-1)} W^{(l)}+b^{(l)}
$$

$$
y^{(l)}=g\left(Z^{(l)}\right)
$$

Where, $y^{(l-1)}$ is stated as the input to layer $l$ and output of prior layers $l-1, Z^{(l)}$ is indicated as the vector of preactivations layers $l, W^{(l)} \in \mathbb{R}^{n_{i} \times n_{0}}$ is stated as the matrix of learnable biases, $l \in[1, \ldots L]$ is represented as the $l^{\text {th }}$ layer, $y^{(L)}$ is indicated as the final layer output, $y^{(l)} \in \mathbb{R}^{n_{0}}$ is specified as the output layer, $g($.$) is represented as the$ nonlinear activation layer, and $y^{(0)}$ is indicated as the input to the model. In this study, ReLU is utilized in the hidden layers for faster learning and computation efficiency related to other activation functions. The output layer uses a softmax non-linearity in order to deliver a probabilistic interpretation of the models output that is mathematically denoted in equation (11).

$$
\operatorname{softmax}\left(Z^{(L)}\right)=
$$

$$
\frac{\exp Z_{k}}{\sum_{k=1}^{K} \exp Z_{k}}
$$

Where, $K$ is indicated as output classes, and the output layer comprises of $K$ number of neurons. The DNN learning is formulated as an optimization issue for minimizing a cost function. In this study, cross entropy loss function is utilized to deal with classification problem, which is defined in equation (12).

$$
C=-\sum_{k=1}^{K} \hat{y}_{k} \log \left(y_{k}^{(L)}\right)
$$

Where, $y^{(L)}$ is denoted as the model output, and $\hat{y}_{k} \in\{0,1\}^{k}$ is indicated as the encoded label.

\section{RESULT AND DISCUSSION}

In this study, MATLAB 2018a environment was used for executing all the experiments with i7 $3.0 \mathrm{GHz}$ processor, 3 TB memory, 8 GB RAM, 2 GB GPU, and windows 10 operating system. Benchmark approaches: For comparing the efficacy of the proposed model, several approaches are considered as the benchmark like sparse representation with DLWH [23], PDCA [24], and 2D reconstructed phase space with LSSVM [25]. Undertaken databases: In this work, the exhaustive simulations are carried out with some of the standard and widely used databases like BB EEG and BU EEG database. The detailed explanation about the undertaken databases are indicated in table 1.

Table 1. Details about the undertaken databases

\begin{tabular}{|c|c|c|c|c|}
\hline Database & Subjects & Total signals & Electrodes & Classes \\
\hline BB EEG & 5 & 7500 & Two & Focal and non-focal \\
\hline BU EEG & 500 & 500 & One & Ictal, interictal, and normal \\
\hline
\end{tabular}

In this scenario, the proposed epilepsy recognition model is quantified by using accuracy, specificity, sensitivity, and PPV with $(\mathrm{k}=10)$ fold cross validation. All the observations in the dataset are eventually utilized for testing and training that is considered as a major benefit of $\mathrm{k}$ fold crossvalidation. The general formula for calculating classification accuracy, specificity, sensitivity, and PPV are defined in the equations (13-16).

$$
\text { Accuracy }=\frac{T P+T N}{F N+T P+F P+T N} \times 100
$$

$$
\text { Specificity }=\frac{T N}{F P+T N} \times 100
$$

$$
\text { Sensitivity }=\frac{T P}{F N+T P} \times 100
$$

$$
P P V=\frac{T P}{F P+T P} \times 100
$$

Where, $T P$ is specified as true positive, $F P$ is represented as false positive, $T N$ is indicated as true negative and $F N$ is stated as false negative.

\subsection{Quantitative investigation on BB EEG dataset}

In this segment, the proposed model performance is investigated by utilizing BB EEG database by means of specificity, accuracy, sensitivity and PPV. Among 7500 available signals, 50 non-focal and focal signals are considered for experimental simulation with $70 \%$ training and $30 \%$ testing of signal. In this scenario, the optimized feature vectors are classified by using different classifiers such as Neural Network (NN), K-Nearest Neighbour (KNN), Multi SVM (MSVM) and DNN for identifying the appropriate classifier for epileptic seizure recognition. By investigating the table 2, the DNN attained better performance related to other classifiers in BB EEG database. From table 2, it is noticed that the DNN classification approach results in maximum of $52.8 \%$ and minimum of $5.3 \%$ improved in accuracy related to other classifiers in epileptic seizure recognition. In addition, the DNN shows superior outcome related to other supervised classifiers (NN, KNN, and MSVM) by means of specificity, PPV, and sensitivity. The graphical evaluation of proposed model with dissimilar classifiers in BB EEG database is indicated in figure 6 .

Published By:

Blue Eyes Intelligence Engineering 
Enhanced firefly optimizer with deep neural network for the detection of epileptic seizures using EEG signals

Table 2. Performance analysis of proposed model with dissimilar classifiers in BB EEG database

\begin{tabular}{|c|c|c|c|c|}
\hline Classifier & Accuracy (\%) & Specificity (\%) & Sensitivity (\%) & PPV (\%) \\
\hline NN & 46 & 55 & 40 & 20 \\
\hline KNN & 92.50 & 95 & 90 & 95.25 \\
\hline MSVM & 93.50 & 87 & 98 & 89.84 \\
\hline DNN & $\mathbf{9 8 . 8 2}$ & $\mathbf{9 8}$ & $\mathbf{9 8 . 9 0}$ & $\mathbf{9 8 . 0 2}$ \\
\hline
\end{tabular}

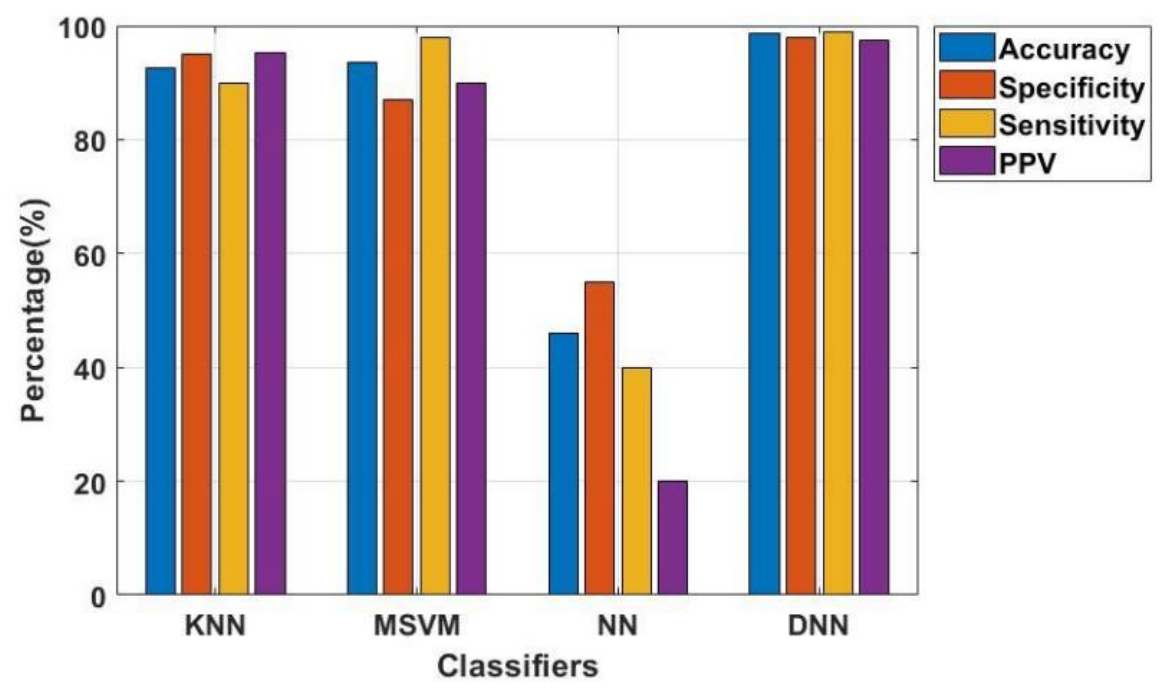

Figure 6. Graphical evaluation of proposed model with dissimilar classifiers in BB EEG database

In addition, the proposed model performance is investigated with dissimilar optimization techniques such as Principal Component Analysis (PCA), particle swarm optimization (PSO), bat algorithm, firefly, and enhanced firefly algorithm. In table 3 , the undertaken optimization techniques are tested with DNN in order to validate the superiority of enhanced firefly-DNN. In this scenario, the undertaken optimization techniques are simulated in the same environment and database for investigating the proposed model (enhanced firefly-DNN) performance. From table 3, it is noticed that the proposed model attained better performance in epileptic seizure recognition related to other techniques by means of specificity, sensitivity, PPV, and accuracy. From the analysis, the proposed model results in maximum of $16.1 \%$ and minimum of $0.12 \%$ improvement in classification accuracy related to other optimization techniques. Similarly, the proposed model (enhanced fireflyDNN) results in maximum of $17.4 \%$ and minimum of $0.03 \%$ improvement in sensitivity related to other techniques. Correspondingly, the proposed model attained an effective performance in epileptic seizure recognition by means of specificity and PPV. Graphical evaluation of proposed model with dissimilar optimizers in BB EEG database is specified in figure 7 .

Table 3. Performance analysis of proposed model with dissimilar optimizers in BB EEG database

\begin{tabular}{|c|c|c|c|c|}
\hline Optimizer & Accuracy (\%) & Specificity (\%) & Sensitivity (\%) & PPV (\%) \\
\hline PCA-DNN & 82.72 & 83.77 & 81.43 & 86.72 \\
\hline PSO-DNN & 95.32 & 91.46 & 95.26 & 94.61 \\
\hline BAT-DNN & 95.62 & 93.21 & 94.35 & 95.07 \\
\hline Firefly-DNN & 98.70 & 98 & 98.87 & 97.36 \\
\hline Enhanced firefly-DNN & $\mathbf{9 8 . 8 2}$ & $\mathbf{9 8}$ & $\mathbf{9 8 . 9 0}$ & $\mathbf{9 8 . 0 2}$ \\
\hline
\end{tabular}

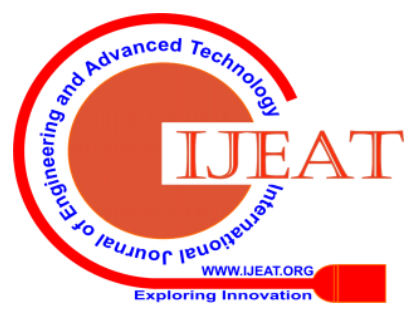




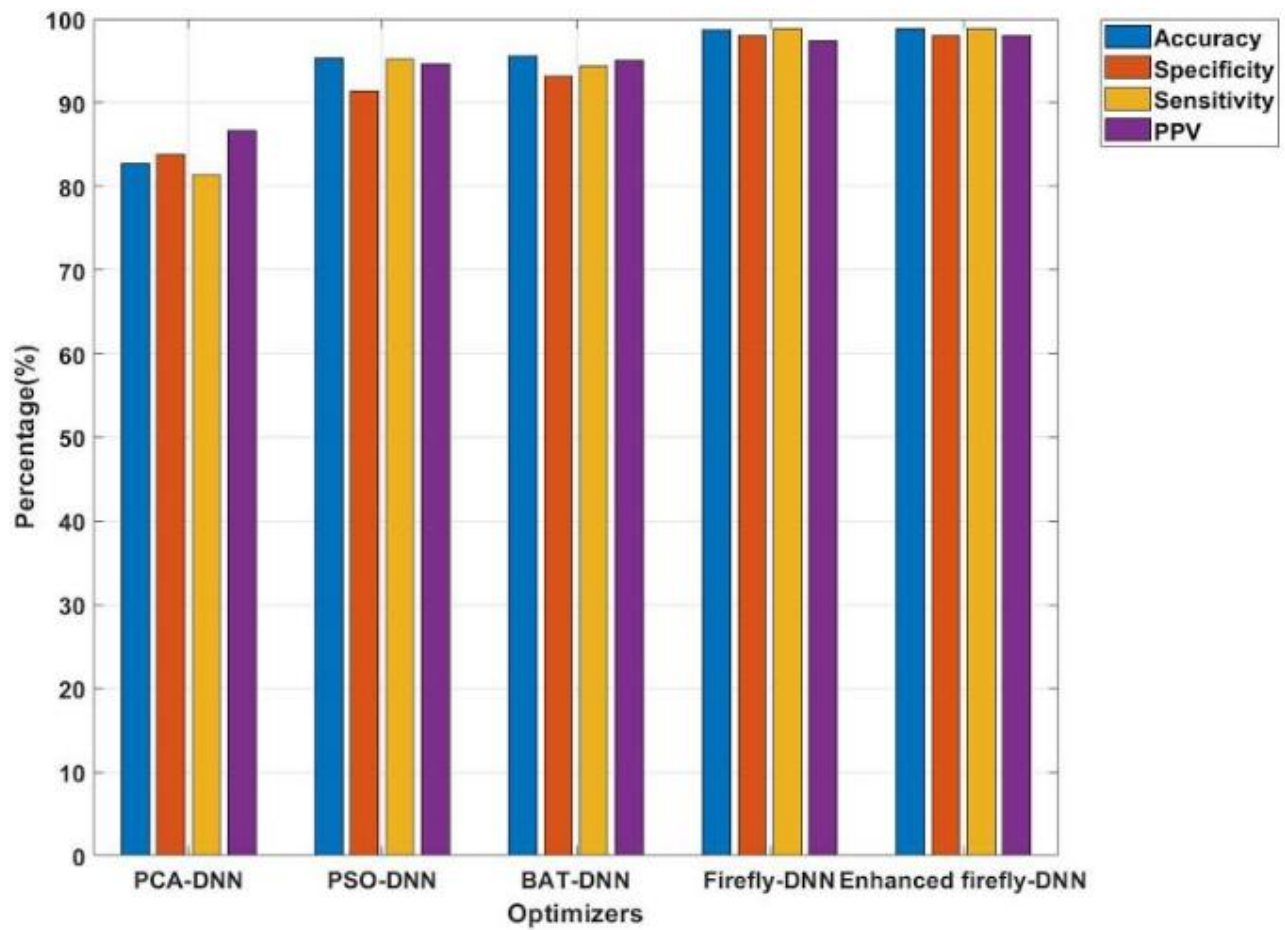

Figure 7. Graphical evaluation of proposed model with dissimilar optimizers in BB EEG database

\subsection{Quantitative investigation on BU EEG dataset}

In this section, the proposed model performance is inspected by using BU EEG dataset by means of specificity, accuracy, sensitivity and PPV. Among 500 signals, 50 ictal, interictal, and normal signals are considered for experimental analysis with $30 \%$ testing and $70 \%$ training of signal. By investigating table 4 , the DNN classification approach attained good performance in epileptic seizure recognition related to other classifiers; NN, KNN, and MSVM. From table 4, it is noticed that the DNN results in maximum of
$65.68 \%$ and minimum of $5.23 \%$ improved in accuracy related to other classification approaches. Likewise, the proposed model achieved an effective performance in epileptic seizure recognition in light of specificity, sensitivity and PPV. The DNN has the capacity to handle multi variety and dimensional data in uncertain and dynamic environment. Due to automatic learning, DNN effectively utilizes the resources and also allows time cycle reduction. The graphical evaluation of proposed model with dissimilar classifiers in BU EEG database is indicated in figure 8.

Table 4. Performance analysis of proposed model with dissimilar classifiers in BU EEG database

\begin{tabular}{|c|c|c|c|c|}
\hline Classifier & Accuracy (\%) & Specificity (\%) & Sensitivity (\%) & PPV (\%) \\
\hline NN & 33.39 & 29 & 89 & 33.33 \\
\hline KNN & 93.33 & 95 & 85.00 & 90 \\
\hline MSVM & 93.84 & 95 & 90 & 89.89 \\
\hline DNN & $\mathbf{9 9 . 0 7}$ & $\mathbf{9 9 . 2 0}$ & $\mathbf{9 8 . 9}$ & $\mathbf{9 8 . 7 5}$ \\
\hline
\end{tabular}

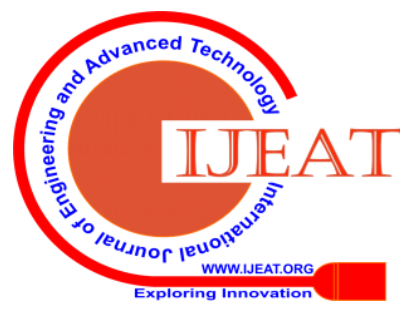


Enhanced firefly optimizer with deep neural network for the detection of epileptic seizures using EEG signals

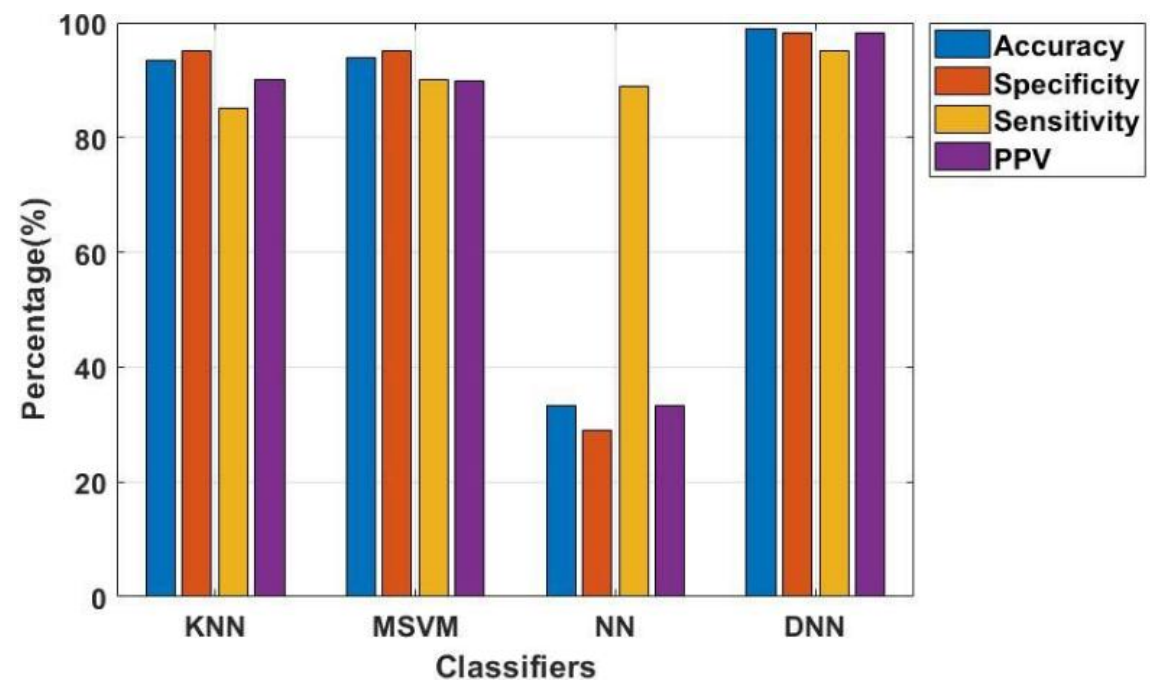

Figure 8. Graphical evaluation of proposed model with dissimilar classifiers in BU EEG database

In table 5, the proposed model performance is investigated with dissimilar optimization techniques like PCA, PSO, bat algorithm, firefly, and enhanced firefly algorithm in BU EEG database. From table 5, it is observed that the proposed model (enhanced firefly-DNN) attained better performance in epileptic seizure recognition related to other optimization techniques by means of specificity, sensitivity, PPV, and accuracy. Hence, the conventional firefly technique is robust and flexible, but it has a major issue of poor exploitation capability that significantly degrades the output solution. To address this concern, a crossover operator of genetic algorithm is included in firefly algorithm for improving the local convergence rate that results in better classification rate. Graphical evaluation of proposed model with dissimilar optimizers in BU EEG database is specified in figure 9.

Table 5. Performance analysis of proposed model with dissimilar optimizers in BU EEG database

\begin{tabular}{|c|c|c|c|c|}
\hline Optimizer & Accuracy (\%) & Specificity (\%) & Sensitivity (\%) & PPV (\%) \\
\hline PCA-DNN & 80.29 & 79.21 & 78.68 & 68.51 \\
\hline PSO-DNN & 87.64 & 85.18 & 87.81 & 75.68 \\
\hline BAT-DNN & 88 & 87.26 & 86.45 & 78.12 \\
\hline Firefly-DNN & 98.99 & 98.32 & 95.07 & 98.13 \\
\hline Enhanced firefly-DNN & $\mathbf{9 9 . 0 7}$ & $\mathbf{9 9 . 2 0}$ & $\mathbf{9 8 . 9}$ & $\mathbf{9 8 . 7 5}$ \\
\hline
\end{tabular}

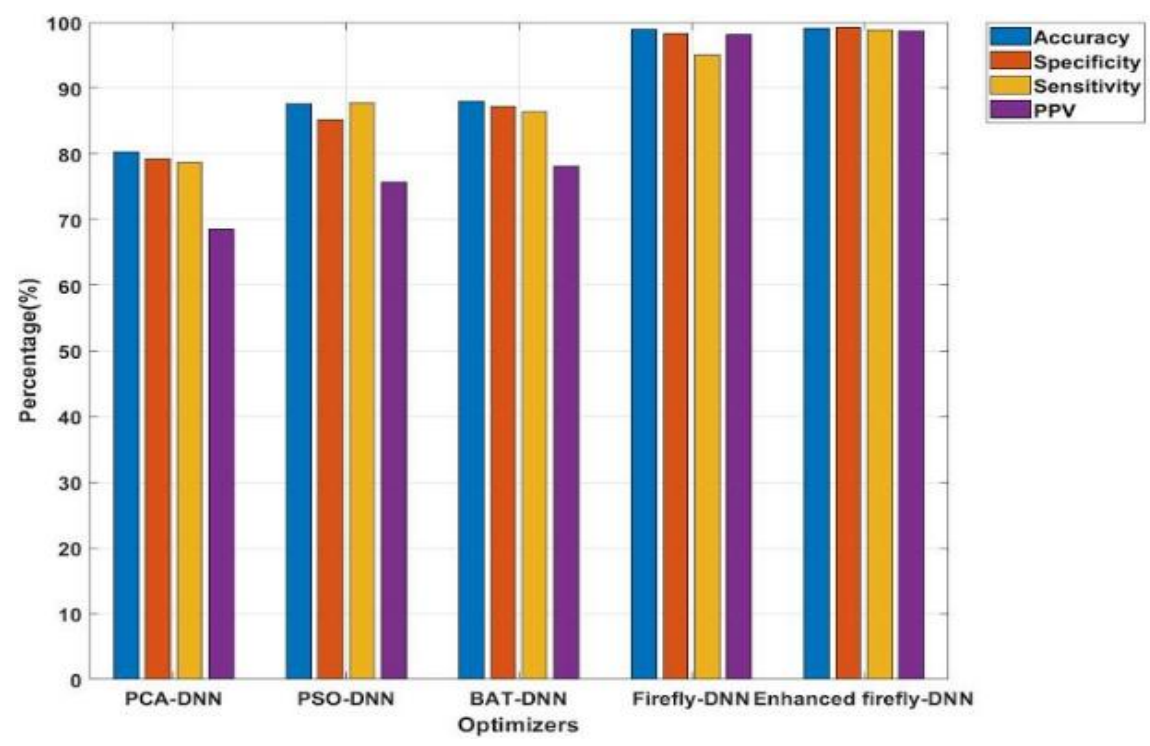

Figure 9. Graphical evaluation of proposed model with dissimilar optimizers in BU EEG database

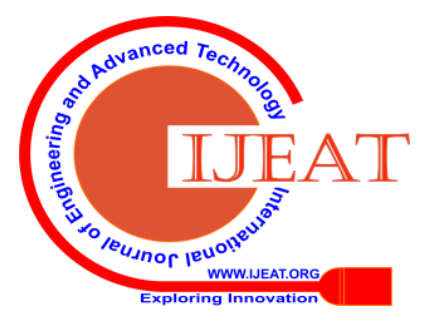




\subsection{Comparative analysis}

In this segment, the comparative investigation about proposed and existing models is indicated in table $7 . \mathrm{H}$. Peng, et al, [23] utilized sparse representation with DLWH for epilepsy recognition. Here, the developed model performance was tested on BU EEG database. From the experimental investigation, the developed model attained $97.60 \%$ of accuracy in classifying interictal, ictal, and normal signals. G. Wang, et al, [24] developed PDCA with multivariate autoregressive features for epilepsy recognition. The developed system almost attained $98.30 \%$ of accuracy in classification. In addition, A. Bhattacharyya, et al, [25] used a $2 \mathrm{~d}$ reconstructed phase space and LS-SVM approach for epilepsy recognition. In this literature, the developed model performance was validated on BB EEG dataset. In the resulting section, the developed model attained $90 \%$ of classification accuracy in epileptic seizure recognition. Related to these existing papers, the proposed model showed good result in epileptic seizure recognition. In BU EEG dataset, the proposed model (Enhanced firefly-DNN) results in maximum of $1.4 \%$ and minimum of $0.77 \%$ improvement in accuracy related to existing research papers. Meanwhile, in BB EEG dataset, the proposed model showed $8.82 \%$ improvement in accuracy related to the existing paper.

Table 6. Comparative investigation

\begin{tabular}{|c|c|c|}
\hline Methods & Dataset & $\begin{array}{c}\text { Accuracy } \\
\text { (\%) }\end{array}$ \\
\hline $\begin{array}{c}\text { Sparse } \\
\text { representation } \\
\text { with DLWH } \\
\text { [23] }\end{array}$ & $\begin{array}{c}\text { BU } \\
\text { EEG }\end{array}$ & 97.6 \\
\hline PDCA [24] & BU & 98.3 \\
\hline $\begin{array}{c}\text { 2D } \\
\text { reconstructed } \\
\text { phase space } \\
\text { with LSSVM } \\
\text { [25] }\end{array}$ & $\begin{array}{c}\text { BEG } \\
\text { EEG }\end{array}$ & 90 \\
& & \\
\hline $\begin{array}{c}\text { Enhanced } \\
\text { firefly-DNN }\end{array}$ & $\begin{array}{c}\text { BU } \\
\text { EEG }\end{array}$ & $\mathbf{9 9 . 0 7}$ \\
\cline { 2 - 3 } & BB & $\mathbf{9 8 . 8 2}$ \\
& EEG & \\
\hline
\end{tabular}

\section{CONCLUSION}

In this research article, a new optimization based model is proposed for enhancing the performance of epilepsy recognition. The proposed model majorly includes three phases such as decomposition, optimization of extracted features, and classification. After signal collection, MVMD is utilized to decompose the EEG signals into time frequency bands. Then, enhanced firefly optimization algorithm is applied to select the active feature vectors, which are classified by employing DNN classifier. In the experimental segment, the proposed model performance is evaluated in light of PPV, accuracy, specificity, and sensitivity. In BU dataset, the proposed model showed maximum of $1.4 \%$ improvement in accuracy compared to the existing research papers. Similarly, in BB dataset, the proposed model showed maximum of $8.82 \%$ improvement in accuracy. In future work, a hybrid decomposition method can be implemented to improve the epilepsy recognition performance.

\section{REFERENCES}

1. S. Janjarasjitt, "Epileptic seizure classifications of single-channel scalp EEG data using wavelet-based features and SVM", Medical \& biological engineering \& computing, vol.55, no.10, pp.1743-1761, 2017.

2. V. Bajaj, and R.B. Pachori, "Epileptic seizure detection based on the instantaneous area of analytic intrinsic mode functions of EEG signals", Biomedical Engineering Letters, vol.3, no.1, pp.17-21, 2013.

3. U. Orhan, M. Hekim, and M. Ozer, "Epileptic seizure detection using probability distribution based on equal frequency discretization", Journal of medical systems, vol.36, no.4, pp.2219-2224, 2012.

4. H.S. Alaei, M.A. Khalilzadeh, and A. Gorji, "Optimal selection of SOP and SPH using fuzzy inference system for on-line epileptic seizure prediction based on EEG phase synchronization", Australasian Physical \& Engineering Sciences in Medicine, vol.42, no.4, pp.1049-1068, 2019.

5. J.G. Bogaarts, E.D. Gommer, D.M.W. Hilkman, V.H.J.M. van Kranen-Mastenbroek, and J.P.H. Reulen, "EEG feature preprocessing for neonatal epileptic seizure detection", Annals of biomedical engineering, vol.42, no.11, pp.2360-2368, 2014.

6. M. Fan, and C.A. Chou, "Detecting abnormal pattern of epileptic seizures via temporal synchronization of EEG signals", IEEE Transactions on Biomedical Engineering, vol.66, no.3, pp.601-608, 2018.

7. K. Samiee, P. Kovacs, and M. Gabbouj, "Epileptic seizure classification of EEG time-series using rational discrete short-time Fourier transform", IEEE transactions on Biomedical Engineering, vol.62, no.2, pp.541-552, 2014.

8. R. Rosas-Romero, E. Guevara, K. Peng, D.K. Nguyen, F. Lesage, P. Pouliot, and W.E. Lima-Saad, "Prediction of epileptic seizures with convolutional neural networks and functional near-infrared spectroscopy signals", Computers in biology and medicine, vol.111, pp.103355, 2019.

9. S. Raghu, N. Sriraam, A.S. Hegde, and P.L. Kubben, "A novel approach for classification of epileptic seizures using matrix determinant", Expert Systems with Applications, vol.127, pp.323-341, 2019.

10. M.S. Islam, A.M. El-Hajj, H. Alawieh, Z. Dawy, N. Abbas, and J. ElImad, "EEG mobility artifact removal for ambulatory epileptic seizure prediction applications", Biomedical Signal Processing and Control, vol.55, pp.101638, 2020

11. M.F. Bari, and S.A. Fattah, "Epileptic seizure detection in EEG signals using normalized IMFs in CEEMDAN domain and quadratic discriminant classifier", Biomedical Signal Processing and Control, vol.58, pp.101833, 2020.

12. F. Stabile, J. van Dijk, C.R. Barnett, and L. De Risio, "Epileptic seizure frequency and semiology in dogs with idiopathic epilepsy after initiation of imepitoin or phenobarbital monotherapy", The Veterinary Journal, vol.249, pp.53-57, 2019.

13. R. Hussein, M. Elgendi, Z.J. Wang, and R.K. Ward, "Robust detection of epileptic seizures based on L1-penalized robust regression of EEG signals", Expert Systems with Applications, vol.104, pp.153-167, 2018.

14. Z.R. Zamir, "Detection of epileptic seizure in EEG signals using linear least squares preprocessing", Computer methods and programs in biomedicine, vol.133, pp.95-109, 2016.

15. X. Fan, N. Gaspard, B. Legros, F. Lucchetti, R. Ercek, and A Nonclercq, "Automated epileptic seizure detection based on break of excitation/inhibition balance", Computers in biology and medicine, vol.107, pp.30-38, 2019.

16. O. Kocadagli, and R. Langari, "Classification of EEG signals for epileptic seizures using hybrid artificial neural networks based wavelet transforms and fuzzy relations", Expert Systems with Applications, vol.88, pp.419-434. 2017.

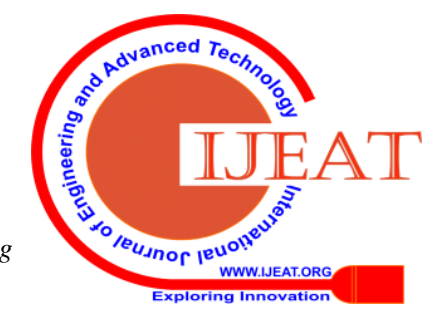


17. Y. Jiang, W. Chen, and M. Li, "Symplectic geometry decompositionbased features for automatic epileptic seizure detection", Computers in biology and medicine, vol.116, pp.103549, 2020.

18. D. Li, Q. Xie, Q. Jin, and K. Hirasawa, "A sequential method using multiplicative extreme learning machine for epileptic seizure detection", Neurocomputing, vol.214, pp.692-707, 2016.

19. H. Kalbkhani, and M.G. Shayesteh, "Stockwell transform for epileptic seizure detection from EEG signals", Biomedical Signal Processing and Control, vol.38, pp.108-118, 2017.

20. K.M. Tsiouris, V.C. Pezoulas, M. Zervakis, S. Konitsiotis, D.D. Koutsouris, and D.I. Fotiadis, "A Long Short-Term Memory deep learning network for the prediction of epileptic seizures using EEG signals", Computers in biology and medicine, vol.99, pp.24-37, 2018.

21. N. Mahmoodian, A. Boese, M. Friebe, and J. Haddadnia, "Epileptic seizure detection using cross-bispectrum of electroencephalogram signal", Seizure, vol.66, pp.4-11, 2019.

22. K. Jindal, R. Upadhyay, and H.S. Singh, "Application of tunable-Q wavelet transform based nonlinear features in epileptic seizure detection", Analog Integrated Circuits and Signal Processing, vol.100, no.2, pp.437-452, 2019.

23. H. Peng, C. Li, J. Chao, T. Wang, C. Zhao, X. Huo, and B. Hu, "A novel automatic classification detection for epileptic seizure based on dictionary learning and sparse representation", Neurocomputing, 2019.

24. G. Wang, Z. Sun, R. Tao, K. Li, G. Bao, and X. Yan, "Epileptic seizure detection based on partial directed coherence analysis", IEEE journal of biomedical and health informatics, vol.20, no.3, pp.873879, 2015.

25. A. Bhattacharyya, M. Sharma, R.B. Pachori, P. Sircar, and U.R. Acharya, "A novel approach for automated detection of focal EEG signals using empirical wavelet transform", Neural Computing and Applications, vol.29, no.8, pp.47-57, 2018.

26. R.G. Andrzejak, K. Schindler, and C. Rummel, "Nonrandomness, nonlinear dependence, and nonstationarity of electroencephalographic recordings from epilepsy patients", Physical Review E, vol.86, no.4, pp.046206, 2012.

27. K. Polat, and S. Güneş, "Artificial immune recognition system with fuzzy resource allocation mechanism classifier, principal component analysis and FFT method based new hybrid automated identification system for classification of EEG signals", Expert Systems with applications, vol.34, no.3, pp.2039-2048, 2008.

28. E.D. Übeyli, and İ. Güler, "Features extracted by eigenvector methods for detecting variability of EEG signals", Pattern Recognition Letters, vol.28, no.5, pp.592-603, 2007.

29. N. ur Rehman, and H. Aftab, "Multivariate Variational Mode Decomposition", IEEE Transactions on Signal Processing, vol.67, no.23, pp.6039-6052, 2019.

\section{Dataset links:}

\section{BB EEG dataset:}

http://ntsa.upf.edu/downloads/andrzejak-rg-schindler-k-rummel-c-2012nonrandomness-nonlinear-dependence-and

BU EEG database: http://epileptologiebonn.de/cms/front_content.php?idcat=193\&lang=3

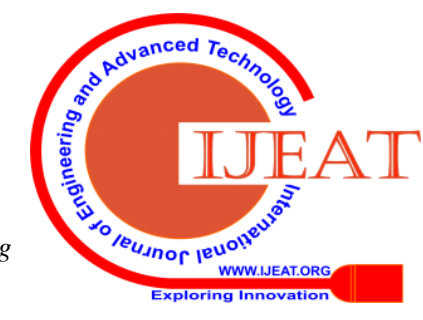

